\title{
Maternal PrEP Use in HIV-Uninfected Pregnant Women in South Africa: Role of Stigma in PrEP Initiation, Retention and Adherence
}

\author{
Alexander Moran ${ }^{1}$ (1) $\cdot$ Nyiko Mashele ${ }^{2} \cdot$ Rufaro Mvududu $^{2} \cdot$ Pamina Gorbach $^{1} \cdot$ Linda-Gail Bekker $^{3}$. \\ Thomas J. Coates ${ }^{4}$. Landon Myer ${ }^{2}$. Dvora Joseph Davey ${ }^{1,2}$
}

Accepted: 9 July 2021 / Published online: 21 July 2021

(c) The Author(s), under exclusive licence to Springer Science+Business Media, LLC, part of Springer Nature 2021

\begin{abstract}
Pregnant women in sub-Saharan Africa are at high risk of HIV acquisition and require effective methods to prevent HIV. In a cohort of pregnant women offered Pre-exposure prophylaxis (PrEP), we evaluate the relationship between internalized and anticipated stigma and PrEP initiation at first antenatal visit, 3-month continuation and adherence using multivariable logistic regression. High internalized and anticipated PrEP stigma are associated with lower PrEP care initiation at first antenatal visit ( $\mathrm{aOR}$ internalized stigma $=0.06 ; 95 \% \mathrm{CI}=0.03-0.11$ and $\mathrm{aOR}$ anticipated stigma $=0.55 ; 95 \% \mathrm{CI}=0.31-1.00)$ compared to women with low reported stigma, after controlling for covariates. Women whose partners have not been tested for HIV or whose serostatus remains unknown have 1.6-times odds of PrEP retention at 3-months compared to women whose partners have been tested $(\mathrm{aOR}=1.60 ; 95 \% \mathrm{CI}=1.02-2.52)$ after adjusting for covariates. PrEP counseling and maternal PrEP interventions must consider individual- and relational-level interventions to overcome anticipated PrEP stigma and other barriers to PrEP initiation and adherence.
\end{abstract}

Keywords PrEP $\cdot$ HIV prophylaxis $\cdot$ Stigma $\cdot$ Pregnancy $\cdot$ South Africa

\section{Introduction}

HIV incidence among pregnant women in South Africa remains high despite successful roll out of interventions to prevent HIV in pregnancy [1-3]. Additionally, seroconversion during pregnancy or the postpartum period continues to contribute to over $30 \%$ of pediatric HIV incidence [4]. Aside from hormonal changes which may affect HIV risk, behavioral changes during pregnancy and the postpartum period remain important determinants of HIV acquisition, including

Alexander Moran

alexmoran@ucla.edu

1 Department of Epidemiology, Fielding School of Public Health, University of California Los Angeles, 615 E Charles Young Drive S, Los Angeles, CA 90095, USA

2 Department of Biostatistics and Epidemiology, School of Public Health and Family Medicine, University of Cape Town, Cape Town, South Africa

3 The Desmond Tutu HIV Centre, University of Cape Town, Cape Town, South Africa

4 David Geffen School of Medicine, University of California Los Angeles, Los Angeles, CA, USA condom use, partner HIV testing and sexual activity [1, 5-8]. These risks are compounded among pregnant Adolescent girls and young women (AGYW) in South Africa, who may have lower HIV care engagement and later, or less frequent, Antenatal care (ANC) attendance [9].

HIV Pre-exposure prophylaxis (PrEP) is a safe, effective and user-controlled HIV prevention method [10-12]. PrEP is effective in preventing HIV transmission in many settings including heterosexual HIV transmission and transmission among men who have sex with men (MSM) [13-16]. PrEP is also safe and effective for cisgender women, as shown in clinical trials including the Partners PrEP study which demonstrated a relative reduction in HIV incidence of $75 \%$ among combined emtricitabine/tenofovir (FTC/TDF) PrEP users compared to placebo [17]. While pregnant women have been excluded from clinical trials of PrEP due to unknown effects of FTC/TDF on pregnancy outcomes, the World health organization (WHO) recommends PrEP use among pregnant and breastfeeding women following recent trials and reviews which suggest that PrEP is safe and effective in preventing HIV among this population [18, 19]. Despite these successes, continuation in PrEP care and adherence to PrEP remain challenges, especially among 
pregnant and postpartum women, among whom emerging pharmacokinetic evidence suggests that blood levels of TDF among African AGYW were about one third lower in pregnancy than in postpartum, indicating the need for daily PrEP adherence to maintain effectiveness against HIV infection [20-22].

There is a dearth of research on stigma and its association with PrEP care initiation, continuation and adherence among pregnant women. Both anticipated and internalized stigma may serve as important barriers to entering PrEP care, continuing in PrEP care and remaining adherent to PrEP among pregnant and postpartum women [23-27]. Anticipated stigma, which describes the expectation of prejudicial or discriminatory behavior because of PrEP use, and internalized stigma, which describes an individual's own belief in the negative ideas associated with PrEP, may act simultaneously or independently [28, 29]. Specifically, anticipated stigma may play an additional role in partner HIV status disclosure, PrEP disclosure and HIV testing history in which people who anticipate stigma may avoid disclosing the use of HIV prevention methods to partners [30].

We conducted this study to understand the role of anticipated and internalized stigma in initiation of PrEP care, continuation in PrEP care and PrEP adherence among pregnant and postpartum women in South Africa. We hypothesize that internalized stigma and anticipated stigma are associated with lower initiation of PrEP care, continuation in PrEP care and PrEP adherence and stand as barriers for pregnant PrEP users. Additionally, we hypothesize that partner HIV serostatus and prior HIV testing are important factors in determining both anticipated stigma and initiation of PrEP care, continuation in PrEP care and PrEP adherence. These findings can inform PrEP delivery strategies, including counseling methods, awareness building in the community, male partner involvement and adherence support strategies.

\section{Methods}

The PrEP-PP (PrEP in Pregnant and Postpartum women) study is an open prospective cohort which enrolls consenting pregnant, HIV-uninfected adolescent girls and women (age $\geq 16$ years) at the first Antenatal care (ANC) visit and follows participants through 12-months post-delivery (Clinical Trial Registry: NCT03902418). The study recruits at one public health clinic in Cape Town, Western Cape, South Africa. Recruitment began in August 2019 and is ongoing $(\mathrm{N}=623)$, with a planned sample size of $\mathrm{N}=1200$ women. The study was approved by the Human Research Ethics Committee at the University of Cape Town (\#297/2018) and by the University of California, Los Angeles Institutional Review Board (IRB\#18-001622). The PrEP-PP study aims to evaluate the distribution of women across the PrEP cascade, including the proportion of women who initiate PrEP among all enrolled, the proportion retained in the PrEP cohort, the proportion who adhere to PrEP using objective Dried blood spot (DBS) measures and self-reported pill count measures. The second aim is to evaluate patient- and provider- level factors associated with the PrEP cascade using quantitative and qualitative approaches.

\section{Study Participants}

Eligible participants provide informed consent to participate in the study. Study eligibility criteria include: (1) Age of at least 16 years, (2) Confirmed HIV-negative serostatus by a 4 th generation antigen/antibody combination HIV test (Abbott Laboratories, Chicago, IL, US) (3) Intention of giving birth in the Midwife obstetrics unit (MOU) of the enrollment facility, (4) Confirmed pregnancy status and (5) Absence of psychiatric or medical contraindications to PrEP. Participants are ineligible if they are concurrently enrolled in another HIV vaccine or prevention trial or if they have any medical contraindications as assessed by the Principal Investigator and study clinicians. Participants who are under 16 or 17 years of age and pregnant are able to consent to participate in the study without parental consent per approval from the University of Cape Town (UCT) IRB [31]. Participants are censored upon HIV seroconversion, pregnancy loss or infant death, migration away from the study area, transfer out of care at the study facility or loss to follow up (defined by not returning to the study for a clinical visit for more than 90 days after unsuccessful participant tracking by the study staff, or if the participant withdraws consent for future visits). Participant tracking efforts include collecting tracing information at the enrollment visit and updating this information at follow up visits, providing reminder calls to participants before scheduled visits, followed by calls and SMSs to participants who miss their visit. If these methods fail, study staff initiate tracing (including home visits) for participants who do not attend their follow up visit within two weeks of the scheduled date. Starting in March 2020, during the government-mandated lockdowns related to the COVID-19 pandemic, phone interviews were offered to participants in place of clinic or home visits [32].

Health care providers at study facilities provide group counseling at baseline, which includes information on HIV testing and counseling, Antiretroviral therapy (ART) for Prevention of mother to child transmission of HIV (PMTCT) and the importance of HIV prevention for women who are HIV-negative. Eligible, consenting participants receive 120 Rand per visit (approximately \$8 USD) in grocery vouchers for their time and effort in the study, as well as remuneration for transportation costs. Participants also receive refreshments (i.e., sandwiches and a cooldrink) on the day of their visit. 


\section{Data Collection}

Following South African HIV testing guidelines, HIV counselors provide all ANC attendees with pre-test counseling for HIV, rapid HIV testing and post-test counseling [33]. Upon confirmation of HIV seronegative status, trained study staff approach women to introduce the HIV prevention study. Upon agreement to participate in the study, the participant consents to screening for study eligibility, which includes a rapid HIV antigen/antibody test and an HBsAg test (Abbott Laboratories). Upon confirmation of eligibility and informed study consent, a trained interviewer conducts a 30 to $45 \mathrm{~min}$ baseline visit survey and records responses in REDCap, a secure, web-based database platform [34, 35]. Participants also receive individual counseling about HIV prevention in pregnancy, including PrEP, along with information on consistent and correct condom use, knowing their partner's HIV status (including referral for male partner or couples HIV testing and counseling), and HIV risk associated with serodiscordance. At baseline, participants self-collect a vaginal swab that is tested for Chlamydia trachomatis (CT), Neisseria gonorrhoeae (NG), and Trichomonas vaginalis (TV) using point of care testing (Cephid, Inc., Sunnyvale, CA, US) and treatment is received as needed during the same visit following National STI Guidelines [36]. Participants who are diagnosed with an STI also receive a partner notification letter for partner STI treatment.

Following the baseline survey, the study interviewer provides information about PrEP and the benefits of taking PrEP. The interviewer then asks the participant if they are interested in starting PrEP, nothing that if they are unsure or uninterested, their response will not impact their study participation. For study participants who consent to taking PrEP, the study nurse draws blood to measure baseline creatinine levels, results for which are confirmed within 24 to $48 \mathrm{~h}$. Upon confirmation that the participant wants to use PrEP, the nurse provides the patient with a one-month supply of Truvada ${ }^{\circledR}$ (tenofovir disoproxil fumarate/emtricitabine [TDF-FTC]) and an invitation card to return in one month for follow up testing (after which participants will receive a three-month prescription to correspond with quarterly study follow-up visits). Participants who do not start PrEP receive an invitation to return in three months for a quarterly study follow-up visit. The entire baseline visit lasts between 60 and $90 \mathrm{~min}$.

After the baseline visit and one-month refill monitoring visit, follow up visits are quarterly and coincide with ANC visits until birth and first postpartum visit. Trained interviewers conduct follow up interviews for all participants. Among PrEP users, follow up visits additionally include adherence counseling for PrEP, a blood draw for Dried blood spot (DBS) testing to measure serum Tenofovir diphosphate (TFV-DP) levels for objective levels of PrEP adherence
[37], and renal function following Southern African PrEP guidelines [38, 39]. Follow-up visits last approximately 30 to $45 \mathrm{~min}$.

\section{Survey Measures}

Survey measures are collected at baseline and follow up visit for all participants (on PrEP and not on PrEP). Survey measures were written in English, translated into isiXhosa, and back translated to English. Survey measures include questions on: (a) Basic demographic information and obstetric history (baseline only), (b) Partner HIV status, (c) Sexual behaviors in the past month and past week (including number of sex partners, type of sex, frequency of sex and condom use), (d) Substance use from the Alcohol use disorders identification test (AUDIT) [40] and Drug use disorders identification test (DUDIT) [41], (e) HIV risk perception, (f) Intimate partner violence (using the WHO IPV scale $[42,43])$, (g) Perceived partner, community and social support for PrEP, (h) PrEP and HIV stigma measures, and (i) For PrEP users only, questions related to PrEP adherence according to self-report (seven-day and 30-day recall) and pill count measures, side effects, adverse events, severe adverse events and birth outcomes (after participants have given birth) at follow-up visits.

\section{Outcome Definition: PrEP Care Initiation, Retention, Continuation and Adherence}

We refer to previous publications of PrEP initiation and continuation in the development of our outcome definitions $[21,44]$. We defined initiation of PrEP care as accepting the initial one-month PrEP prescription at baseline. Participants who initiate PrEP care at baseline are invited to return for a one-month follow-up visit to monitor adherence and receive a prescription for a two-month supply of PrEP. PrEP care retention was measured at the three-month follow up visit, in which retention was defined by returning for the first quarterly follow up visit, excluding participants who never started PrEP or had been censored prior to the three-month follow up visit. We defined PrEP care continuation as having returned for the first quarterly follow up visit and having received a PrEP prescription both at the baseline visit and at the one-month follow up visit. Among those who continued in PrEP care, self-reported PrEP adherence was measured using the 30-day recall self-report measure, in which good adherence was defined by reporting taking at least 25 of the last 30 doses preceding the three-month follow up visit (Fig. 1). In sensitivity analyses, we did not find differences between the 7-day and 30-day self-report adherence measure, and in the absence of available DBS TFV-DP results, we report the 30-day self-report measure in favor of the 30-day 


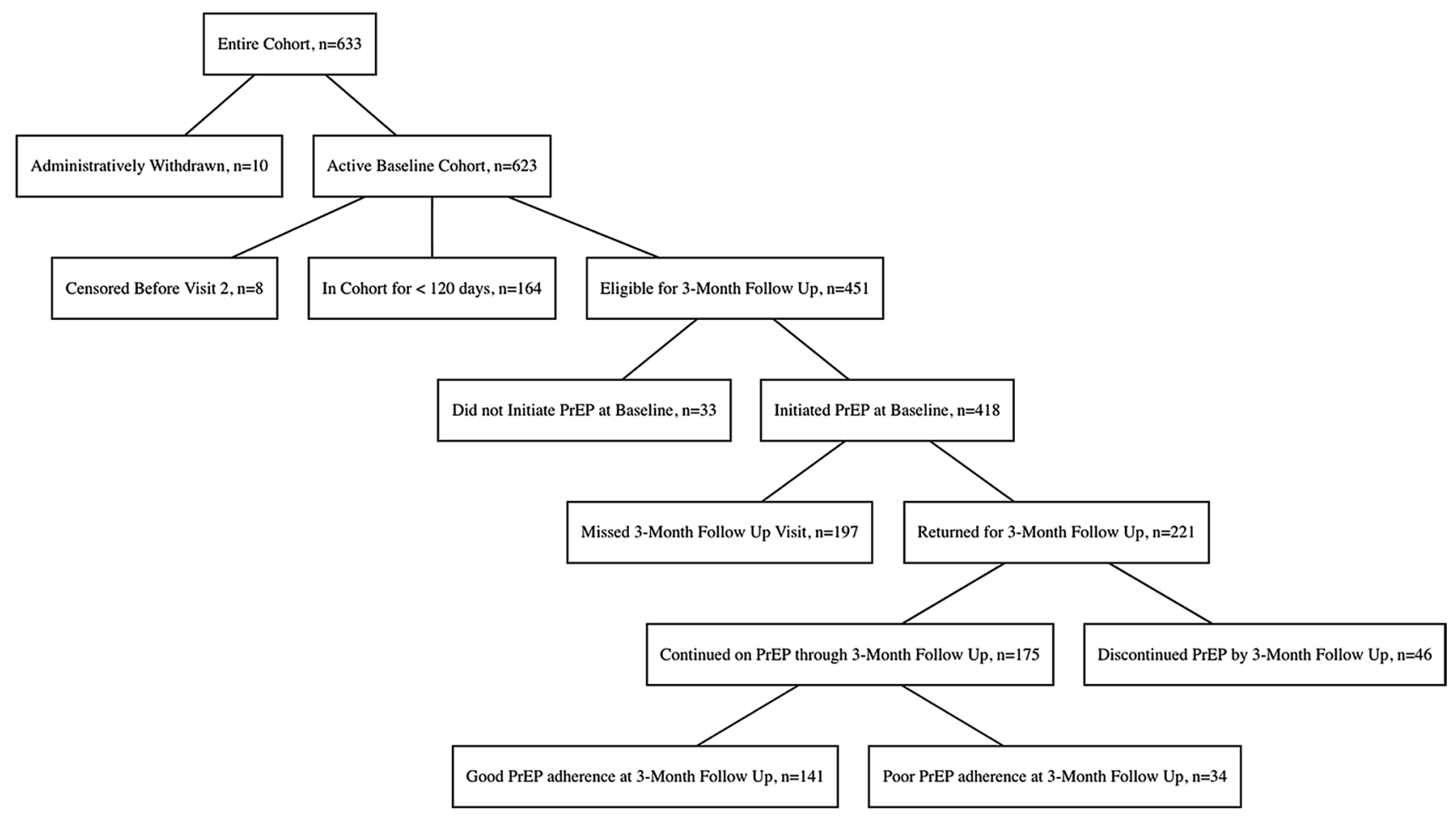

Fig. 1 PrEP-PP cohort and analysis sample, 9-September 2020

pill count measure, which had high missingness and which had lower agreement with both self-report measures.

\section{PrEP Stigma Scales}

We performed an exploratory factor analysis to define two PrEP stigma scales: internalized PrEP stigma and anticipated PrEP stigma. We examined these factors as two separate scales to understand the independent relationships between internalized PrEP stigma and anticipated PrEP stigma, which may function differently in the context of partner HIV testing. Scale measures were dichotomized to high and low stigma, where high stigma indicates that a participant reported a summary stigma score above the median (median $=3$, range $=0-12$ for both internalized PrEP stigma and anticipated PrEP stigma).

We measured stigma with seven questions, of which six questions loaded into two separate factors (internalized PrEP stigma: $\alpha=0.811$; anticipated PrEP stigma: $\alpha=0.777$ ). Individual factor loadings are presented in Table 1 along with the questions used to construct each factor. These stigma questions were chosen based on PrEP stigma questions targeted toward pregnant and postpartum women in the IMPAACT 2009 study [45]. Approximately 10\% of participants in
Table 1 Factor loadings for two stigma scales

\begin{tabular}{lcc}
\hline Survey question & Factor loading & $\alpha$ \\
\hline Factor 1: Internalized PrEP stigma (sample units =623) & 0.811 \\
I feel ashamed of using PrEP & 0.824 & \\
I feel embarrassed about using PrEP & 0.859 \\
$\quad$ I do not feel empowered to use PrEP & 0.556 & 0.777 \\
Factor 2: Anticipated PrEP stigma (sample units=623) & 0.979 \\
I think people will give me a hard time (such as make fun of me, or talk & \\
$\quad$ badly about me) if I tell them I am taking PrEP & 0.721 \\
I think people will judge me negatively if I take PrEP & 0.424 \\
$\quad$ I think I am at greater risk for physical violence or rape if I take PrEP & \\
Eliminated & -0.223 \\
People will think I am behaving responsibly by taking PrEP & \\
\hline
\end{tabular}


the initiation sample $(n=63), 5 \%$ in the retention sample $(n=21), 3 \%$ in the continuation sample $(n=7)$ and $3 \%$ in the adherence sample $(n=5)$ reported high internalized stigma. Prevalence of anticipated stigma was higher, with $26 \%(n=162)$ of participants in the initiation sample, $23 \%$ $(n=98)$ in the retention sample, $18 \%(n=40)$ in the continuation sample and $18 \%(n=31)$ the adherence sample reporting high anticipated stigma.

\section{Statistical Analyses}

As of 9-September 2020, the study had recruited $n=633$ participants, of whom 10 were administratively withdrawn because they were ineligible after consenting. We considered the remaining $n=623$ participants for the PrEP care initiation outcome. Among these, $n=8$ participants were censored before the three-month quarterly follow up visit (visit 2), $\mathrm{n}=164$ had been in the cohort for under 120 days without having completed a three-month follow up visit and $\mathrm{n}=33$ did not start PrEP at baseline; these participants were excluded from PrEP care retention, continuation and adherence outcomes. Among the $n=418$ participants on PrEP who had been in the cohort for at least 120 days, we considered all for the PrEP care retention outcome. We measured PrEP care continuation (receiving a PrEP prescription at both baseline and the one-month follow up visit) among the $\mathrm{n}=221$ participants who attended the first quarterly follow up visit. We finally measured adherence among the $n=175$ participants who continued on PrEP through the first quarterly follow up visit (Fig. 1).

We present distribution of PrEP care initiation, retention and adherence including counts and percentages for categorical variables and medians and Interquartile ranges (IQRs) for continuous variables. We note the sample size for each characteristic presented to highlight any missingness. Missingness was generally low; all participants in the baseline cohort had an outcome for PrEP care initiation, all eligible for initiation had an outcome for PrEP care retention at three months and all eligible for retention had an outcome for PrEP adherence by self-report. Certain demographic variables had missingness (up to $3 \%$, for sex frequency among those in the active baseline cohort and less for all other variables) but we did not impute for data completion. We present demographic characteristics including age, education, income, household size including children, number of living children and marital status. We also include gestational age in weeks at baseline, gravidity (number of prior pregnancies), pregnancy intention and feelings on having a baby. We present HIV risk and sexual behavior data, including partner HIV testing behaviors, partner HIV status, HIV risk perception, sex frequency in the past three months and number of sexual partners during pregnancy. We present self-reported stigma characteristics including internalized
PrEP stigma and anticipated PrEP stigma and psychosocial factors including depression (measured through the Edinburgh postnatal depression scale [EPDS]) [46], concern about sexual violence or rape and IPV experience (any IPV and emotional, physical or sexual IPV). Finally, we present selected substance use characteristics including any drug or alcohol use in the last year before pregnancy using the AUDIT and DUDIT scales.

We assessed potential confounders with Directed acyclic graphs (DAGs) (Supplemental Material). We examined crude associations between possible confounders (gestational age at baseline, education level, gravidity, partner HIV testing, STI at baseline), exposures (internalized PrEP stigma and anticipated PrEP stigma) and outcomes of interest ((1) PrEP care initiation at baseline, (2) PrEP care retention at three months, (3) PrEP care continuation at three months and (4) Self-reported PrEP adherence at three months) in separate models using logistic regression. We chose these variables based on formative research on PrEP use among pregnant women in South Africa [5, 6]. We considered exact logistic regression but did not find significant differences in the results. We hypothesized that internalized stigma and anticipated stigma are associated with lower PrEP initiation, retention in PrEP care, continuation in PrEP care and PrEP adherence and stand as barriers for pregnant PrEP users. We also hypothesized that partner HIV testing is an important factor in determining both anticipated stigma and PrEP care initiation, care retention, care continuation and adherence. We constructed two separate multivariable logistic regression models: one for internalized PrEP stigma (which controlled for gestational age, education level and gravidity) and one for anticipated PrEP stigma (which controlled for gestational age, education level, gravidity and partner HIV testing) and tested these models for the first three outcomes of interest. For the fourth outcome of interest (PrEP adherence at three months) we also controlled for STI at baseline in both the internalized PrEP stigma model and the anticipated PrEP stigma model. All statistical analyses were conducted with R v4.0 (Vienna, Austria) [47].

\section{Results}

\section{Descriptive Analyses of Study Baseline}

We enrolled and followed 623 eligible pregnant women at their first antenatal care visit. Median age in years at baseline was 25 (IQR: 22-30) and median gestational age at baseline was 21 (IQR: 14-29). Half of participants had less than Grade 12 education $(n=313,50 \%)$. Overall, $19 \%$ $(n=121)$ were married and 35\% $(n=218)$ were cohabiting at baseline, $8 \%(\mathrm{n}=50)$ reported not being in a relationship. Median gravidity was two (IQR: $1-3)$ and $35 \%(n=218)$ 
were primigravida. Most participants did not intend to get pregnant or changed their intention $(n=428,69 \%)$. Over one-quarter of participants reported that their partner had not tested for HIV, or that they did not know their partner's serostatus $(n=170,27 \%)$. Reported HIV seropositivity among partners was low ( $\mathrm{n}=8,<2 \%$ for all women). Nearly all participants had at least one sexual partner during pregnancy $(\mathrm{n}=605,97 \%)$ and over half of participants in all samples reported two to four sexual events per month in the last three months. STI prevalence of CT, NG and/or TV was 35\% $(n=216)$ at first antenatal visit. Reported depression (EPDS score $\geq 13)$ was $7.2 \%(n=45)$ at baseline. Half of participants reported no concern about sexual violence or rape in the next three months, but one-third $(n=205,33 \%)$ reported that they were "very concerned". About one tenth of participants $(n=75,12 \%)$ reported any kind of IPV in the past year. Almost half of participants $(n=292,47 \%)$ reported alcohol and/or drug use in the last 12 months (Table 2).

\section{PrEP Care Initiation at Baseline}

Ninety-one percent $(91 \%, n=570)$ of $n=623$ pregnant women initiated PrEP care at baseline. Cohabitation was significantly higher in the initiation sample among those in PrEP care ( $36 \%$ vs. $\left.21 \% ; \chi^{2}=4.500 ; \mathrm{p}=0.034\right)$. Those who did not initiate PrEP care at baseline reported higher internalized stigma (53\% vs. $\left.6.1 \% ; \chi^{2}=111.21 ; \mathrm{p}<0.001\right)$ and higher anticipated stigma $\left(38 \%\right.$ vs. $25 \% ; \chi^{2}=3.505$; $\mathrm{p}=0.061$ ). (Table 2).

\section{PrEP Care Retention at Three Months}

Among the $n=570$ pregnant women who initiated PrEP care at baseline, $n=418$ were eligible for a three-month follow up visit. By study analysis date, 53\% $(\mathrm{n}=221)$ returned for a three-month follow up visit for PrEP care retention. Fewer participants who returned for the 3-month visit reported an HIV negative partner compared to HIV positive or unknown status $\left(67 \%\right.$ vs. $\left.78 \% ; \chi^{2}=8.971 ; \mathrm{p}=0.007\right)$ in comparison to those who missed their visit or did not return. Baseline anticipated PrEP stigma was higher among those who missed or did not return for their 3-month visit, compared to those who returned ( $29 \%$ vs. $\left.18 \% ; \chi^{2}=6.847 ; p=0.009\right)$ (Table 2 ).

\section{PrEP Continuation at Three Months}

Among the $\mathrm{n}=221$ women who were retained in care at three months, $79 \%(n=175)$ continued in PrEP care through the first quarterly follow up visit. There were no differences in demographics when comparing those who continued in PrEP care with those who did not continue in PrEP care among those who returned for the 3-month visit, so we did not tabulate these results.

\section{Self-Reported PrEP Adherence at Three Months}

Among the $\mathrm{n}=175$ women who continued in PrEP care through the first quarterly follow up visit, $81 \%(n=141)$ reported good adherence with a 30-day recall self-report measure. Poorly adherent participants in the adherence sample reported gestational age of at least 20 weeks at study entry more commonly compared to those with good adherence ( $70 \%$ vs. $\left.50 \% ; \chi^{2}=4.027 ; \mathrm{p}=0.045\right)$ and had significantly higher baseline STI prevalence compared to those with good adherence ( $65 \%$ vs $\left.30 \% ; \chi^{2}=12.305 ; \mathrm{p}<0.001\right)$ (Table 2).

\section{Factors Independently Associated with PrEP Initiation, Continuation and Adherence}

Reported internalized stigma was associated with lower odds of PrEP initiation at baseline (aOR: 0.06, 95\% CI: 0.03-0.11) after controlling for gravidity, education and gestational age at baseline. Additionally, anticipated stigma was associated with lower odds of PrEP initiation at baseline (aOR: 0.55, 95\% CI: 0.31-1.00) after controlling for gravidity, education, gestational age at baseline and partner HIV testing (Table 3).

Anticipated stigma was associated with lower odds of returning for three-month study follow up and PrEP prescription among those who initiated PrEP at baseline (aOR: 0.53 , 95\% CI: $0.33-0.84$ ) adjusting for covariates. Having a partner who had not tested for HIV or whose HIV serostatus was unknown were associated with higher odds of returning for the three-month follow up visit (aOR: 1.60, 95\% CI: 1.02-2.52) after controlling for gravidity, education, gestational age at baseline (Table 3). We did not find any association between those who continued in PrEP care at three-month follow up compared to those who did not continue in PrEP care.

Among those who continued in PrEP care at three-month follow up, neither internalized stigma nor anticipated stigma were associated with higher adherence measured by selfreport. Less than Grade 12 education was associated with higher adherence measured by self-report in the internalized stigma model (aOR: 2.37, 95\% CI: 1.03-5.68) and the anticipated stigma model (aOR: 2.57, 95\% CI: 1.11-6.26). Additionally, we found that participants who entered the study with gestational age of at least 20 weeks were at lower odds of good adherence in both the internalized stigma model (aOR: $0.35,95 \%$ CI: $0.14-0.81$ ) and the anticipated stigma model (aOR: 0.34, 95\% CI: 0.14-0.80). Finally, women diagnosed with a STI at baseline had lower odds of good adherence compared to women without an STI in both the internalized stigma model (aOR: 0.20, 95\% CI: 0.09-0.44) and the anticipated stigma model (aOR: $0.19,95 \% \mathrm{CI}$ : $0.08-0.44$ ) (Table 3). 


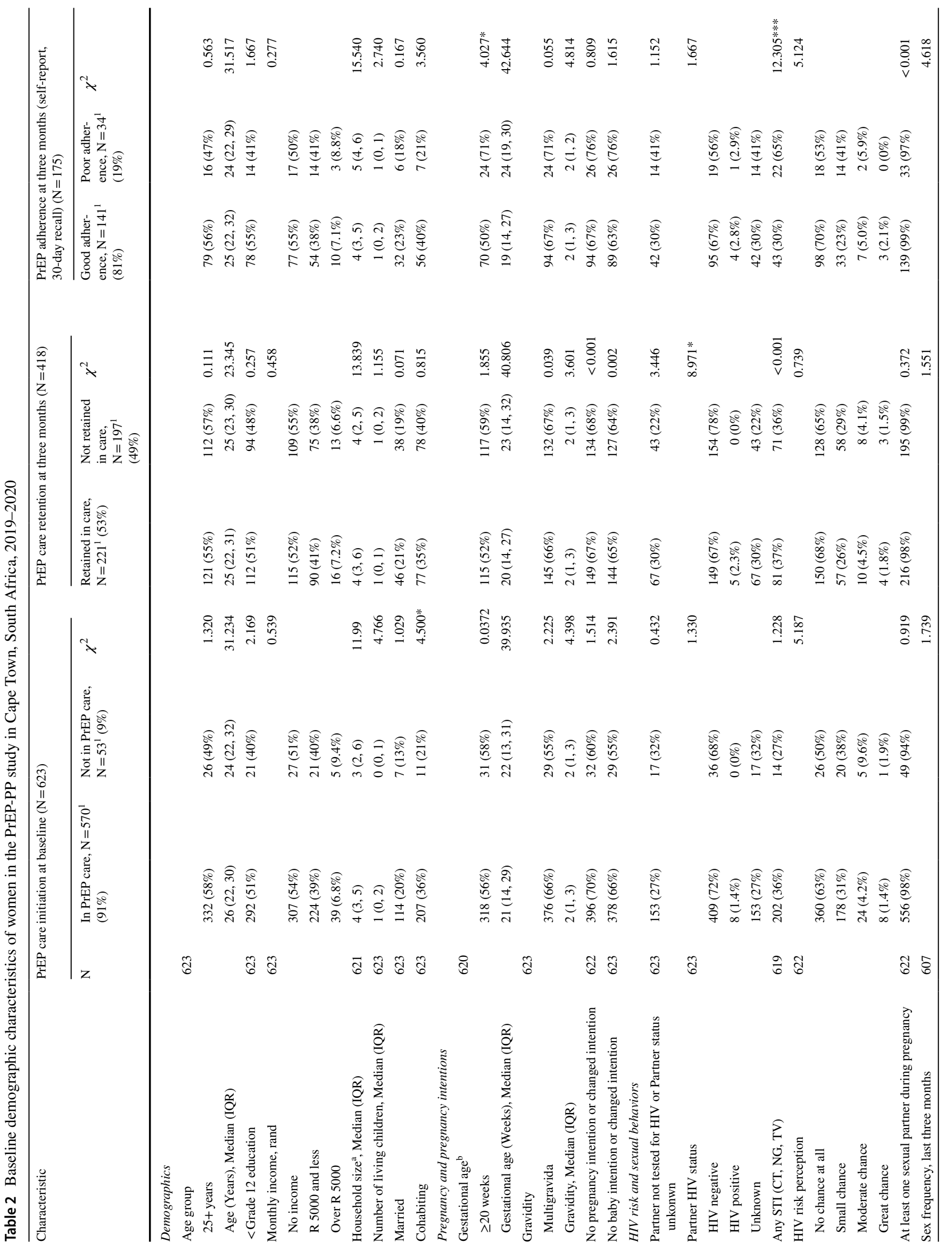




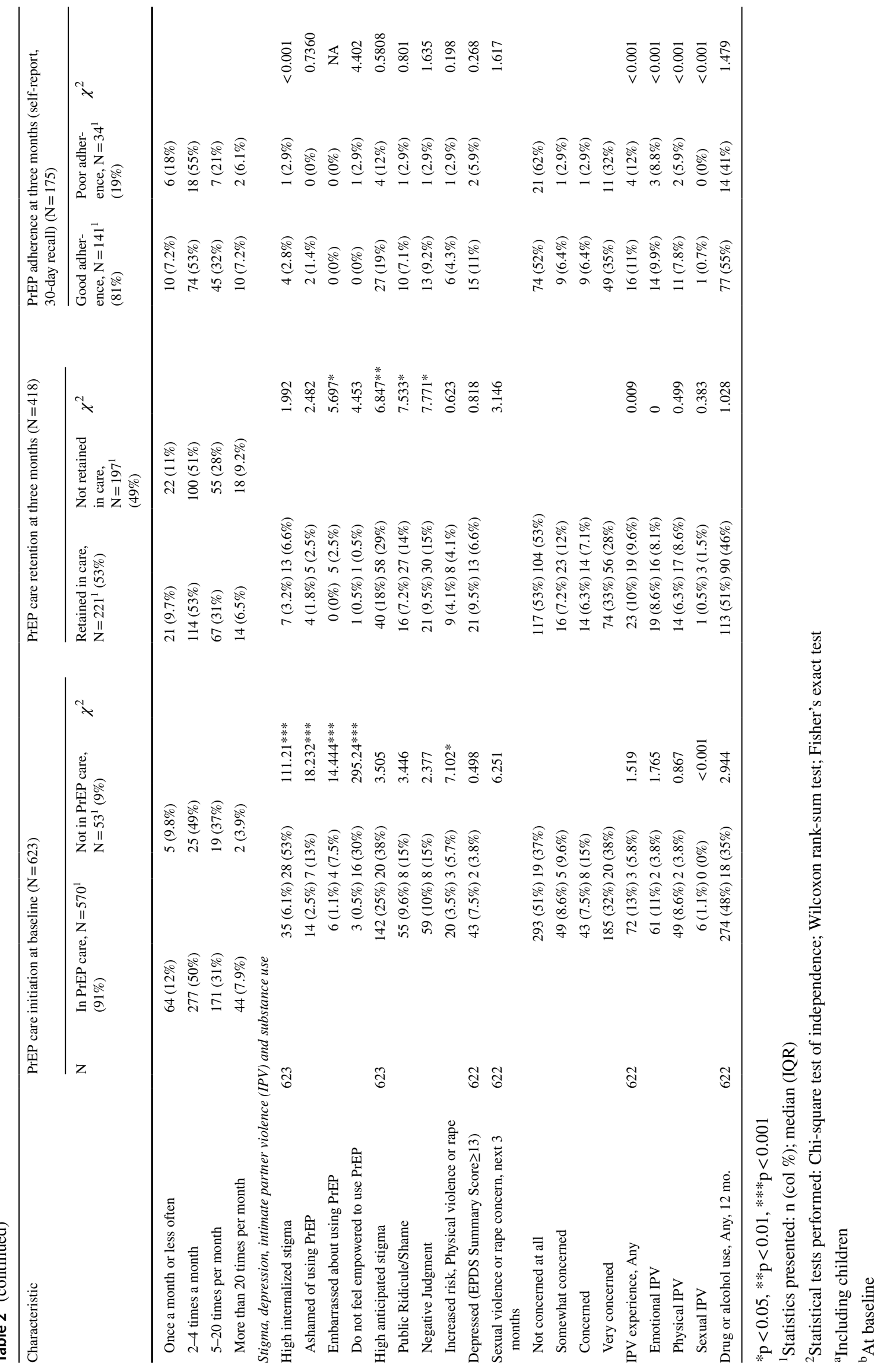




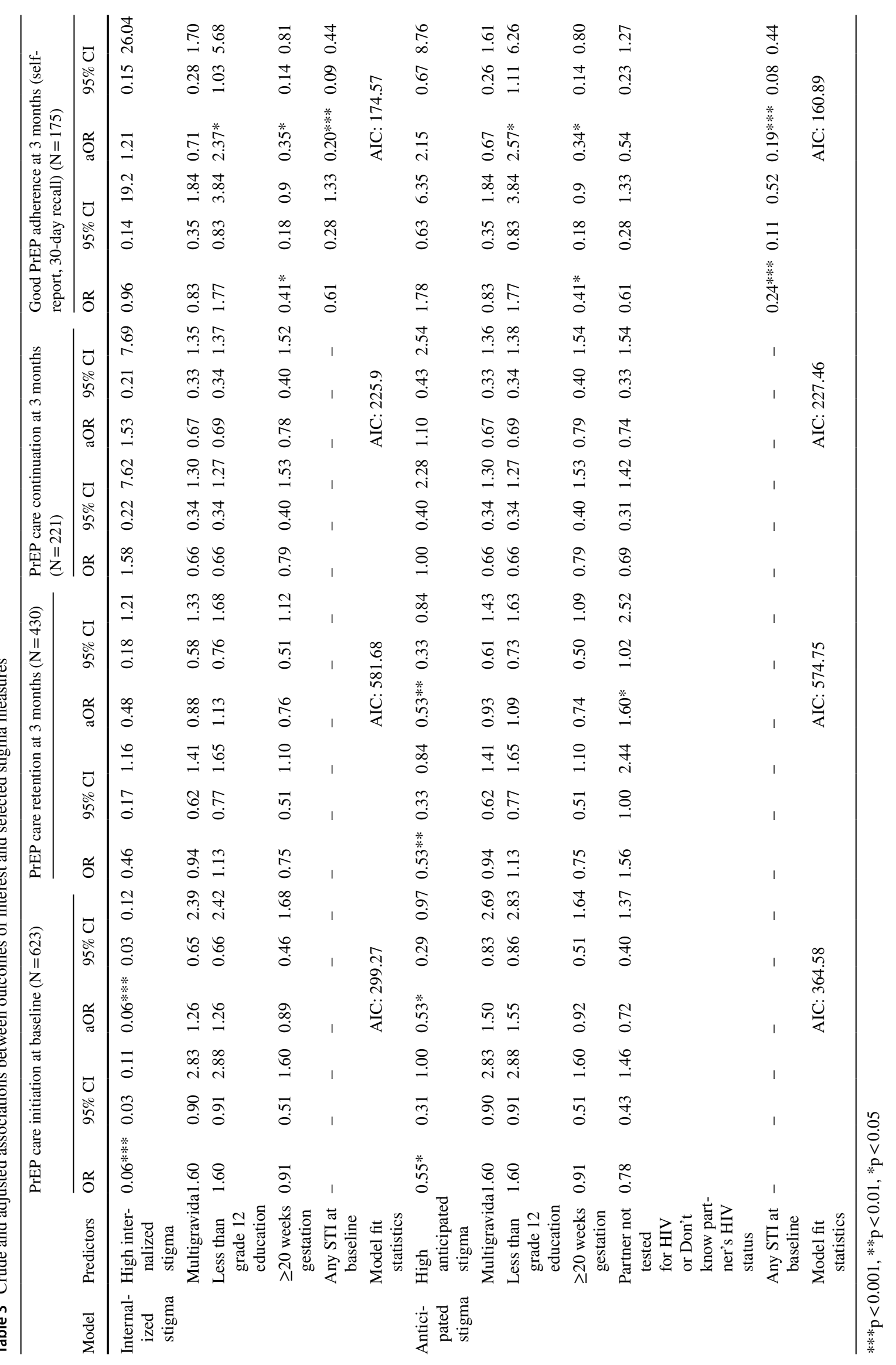




\section{Discussion}

We identified strong relationships between stigma and PrEP care initiation at baseline and PrEP care retention at three-months in a cohort of pregnant and postpartum women. We also identified an association between limited knowledge of partner serostatus and PrEP retention, and between both STI at baseline and gestational age at baseline and PrEP adherence, controlling for PrEP stigma. Specifically, we found that high PrEP internalized stigma was independently associated with lower odds of PrEP care initiation, and that high anticipated PrEP stigma was independently associated both with lower odds of PrEP care initiation at baseline and with lower odds of PrEP care retention at three months. Additionally, PrEP users who did not know their partner's serostatus prior to their first antenatal visit were at lower odds of returning for PrEP follow up at three months after controlling for anticipated PrEP stigma. Finally, women who had an STI at baseline or who entered the study with gestational age of at least 20 weeks were at lower odds of good PrEP adherence at three months after controlling for either internalized or anticipated PrEP stigma.

The results in this study build on previous evaluations of stigma and PrEP initiation, retention, continuation and adherence in South Africa and elsewhere, in which stigma was a driver of PrEP discontinuation among women, MSM and sex workers [8, 23, 26, 48]. While other efforts have categorized stigma as a barrier to PrEP initiation, retention, continuation and adherence, this study further describes internalized stigma and anticipated stigma as individual-level and relational-level barriers with distinct drivers and programmatic solutions. Prior studies demonstrated that stigma associated with the PrEP occasionally led to conflict with male partners as well as PrEP discontinuation or study termination. Corneli et al. found that while participants reported that ART was potent and beneficial for HIV-infected individuals, they were regarded as potentially harmful when taken by HIV-negative individuals, suggesting that improved messaging on the nature of PrEP and other preventive medications could reduce stigma surrounding taking pills [23].

Based on our results, internalized stigma is associated with lower PrEP care initiation in pregnant women, while anticipated stigma is associated with lower PrEP care initiation and retention in PrEP care. In contrast, HIV stigma measures generally showed no association between groups at initiation, retention or continuation. These findings highlight important differences in characterizing stigma among pregnant and postpartum women in South Africa. First, internalized PrEP stigma describes an individual's perception of their own PrEP use as negative or shameful [28]. The relationship between high internalized stigma and lower PrEP initiation indicates that the barrier imposed by internalized stigma is highest at PrEP initiation and underscores the need for individual-level stigma reduction strategies. Second, anticipated stigma focuses on a person's expectation of discrimination or prejudice because of their PrEP use [28]. The relationships between high anticipated stigma and both lower PrEP initiation and retention indicate that anticipated stigma serves as a continuous barrier for PrEP use which requires a different set of interventions outside of internalized stigma mitigation.

Notably, neither internalized nor anticipated stigma was associated with PrEP adherence. Descriptive analyses revealed that a higher proportion of participants who did not initiate PrEP care and who were not retained in PrEP care reported high internalized and anticipated stigma than those who did initiate PrEP care and those who were retained in PrEP care. These results suggest that the most highly stigmatized participants did not engage in PrEP care or were not retained in PrEP care. In contrast, the PrEP adherence outcome was measured only among participants who had continued in PrEP care for at least three months, having returned to the one-month refill visit and three month follow up visit, filling a PrEP prescription at each visit. The group eligible for the adherence outcome may have overcome certain barriers not overcome by the more highly stigmatized participants in the PrEP care initiation and PrEP care retention outcomes who did not initiate PrEP care and who were not retained in PrEP care. Thus, our measure of PrEP adherence may be biased towards women who did not experience or anticipate stigma in pregnancy in the first three-months in PrEP care. Additionally, there are other risk factors that were associated with sub-optimal PrEP use including presenting late for first antenatal care visit ( $>20$ weeks), low educational attainment, and STI diagnosis at baseline. Reaching these participants at high risk for HIV is essential to preventing HIV acquisition in pregnancy and postpartum periods and will require interventions such as flexible, differentiated care models that require less frequent clinic visits for prescriptions, HIV self-testing, and community or home delivery of PrEP [21, 49, 50].

Our findings help to better understand barriers to PrEP use among pregnant and postpartum women. Pregnant women in this sample reported similar experiences as AGYW in the HPTN 082 study among African AGYW, including perceived stigma and external stigma based on presumed HIV positive status because of PrEP use [48]. We recommend including enhanced counselling around PrEP use disclosure among family and partners and adherence clubs or PrEP ambassadors in antenatal and postnatal services to address anticipated and realized stigma. Disclosure of PrEP use to partners and family members may also help to reduce levels of internalized and anticipated stigma [51]. 
For example, having a partner who had not been tested for HIV or whose HIV status was unknown was associated with lower odds of retention. Previous studies have identified that home-based HIV testing is a cost-effective intervention associated with higher uptake of couples testing, higher uptake of male partner testing and improved HIV status disclosure [49-51]. As high anticipated stigma is associated with lower PrEP initiation and retention, interventions which aim to engage male partners in HIV prevention through partner testing and counseling, awareness building and adherence support could reduce the consistent barrier faced by pregnant and postpartum women who anticipate stigma or limited support from partners and significant others.

This study is one of the first to describe stigma in a cohort of pregnant and postpartum women on PrEP in South Africa. There is little existing research on PrEP stigma among pregnant women and even fewer quantitative studies among these. Additionally, our validated scales for internalized PrEP stigma and anticipated PrEP stigma show high internal consistency and reliability for continued measurement and monitoring of stigma among pregnant and postpartum women in South Africa.

Despite these strengths, this study suffers from certain limitations; namely, its cross-sectional nature and selfreported adherence outcome. These cross-sectional associations indicate that PrEP retention at three months is lower among women with high anticipated stigma compared to those with low anticipated stigma, but future longitudinal analyses will be important to better understand these relationships over time. Given that this was a secondary analysis of available data and that the PrEP-PP study was not powered to detect associations between internalized stigma and PrEP use, the analysis may suffer from insufficient power. Additionally, PrEP adherence was self-reported and may be over reported due to social desirability bias or recall error. We will continue to assess the true levels of adherence from TFV-DP DBS in future analyses. Finally, the coronavirus disease 2019 (COVID-19) pandemic led to travel restrictions and government lockdown protocols which affected study retention due to site closures $[32,49,52]$.

\section{Conclusion}

PrEP stands as an effective, safe strategy for primary HIV prevention among pregnant and postpartum women [11]. Aside from programmatic and clinical barriers to optimize maternal PrEP use, behavioral factors act as important determinants of PrEP use and may act at both the individual and relational levels [24, 50]. Effective stigma mitigation must consider the level at which stigma originates (internalized, at the individual level or anticipated, at the relational and individual levels) to effectively reduce barriers for effective
PrEP initiation and use. Improved awareness building among PrEP users and in the broader community can work to improve overall knowledge of PrEP and its benefits. Importantly, solutions which involve male partners - including HIV self-testing for partners, couples counseling and testing, and support in disclosing PrEP use by pregnant women - may be effective to reduce ongoing barriers to optimal PrEP use presented by anticipated PrEP stigma.

Supplementary Information The online version contains supplementary material available at https://doi.org/10.1007/s10461-021-03374-x.

Acknowledgements We would like to thank our study participants, PrEP-PP study staff and the City of Cape Town Department of Health staff. DJD received funding from Fogarty International Center and National Institute of Health (K01TW011187), TC and LM received funding from National Institute of Mental Health (R01MH116771). The authors have no conflicts of interest to declare that are relevant to the content of this article.

Author Contributions DJD: overall technical guidance, writing support and analytical support. LM, TC, LB, PG: overall technical guidance, review and specific technical inputs. RM, NM: analytical support, review and statistical inputs. AM: initial draft, initial statistical analysis and review/revisions. All authors reviewed the final version before submission.

Funding DJD received funding from Fogarty International Center and National Institute of Health (K01TW011187), TC and LM received funding from National Institute of Mental Health (R01MH116771).

\section{Declarations}

Conflict of interest The authors have no conflicts of interest to declare that are relevant to the content of this article.

Ethical Approval The PrEP-PP (PrEP in Pregnant and Postpartum women) study is an open prospective cohort which enrolls consenting pregnant, HIV-uninfected adolescent girls and women (age $\geq 16$ years) at the first Antenatal care (ANC) visit and follows participants through 12-months post-delivery (Clinical Trial Registry: NCT03902418). The study was approved by the Human Research Ethics Committee at the University of Cape Town (\#297/2018) and by the University of California, Los Angeles Institutional Review Board (IRB\#18-001622).

Consent to Participate Eligible participants provide informed consent to participate in the study, which can be rescinded at any point during the study.

\section{References}

1. Dinh T-H, Delaney KP, Goga A, Jackson D, Lombard C, Woldesenbet $\mathrm{S}$, et al. Impact of maternal HIV seroconversion during pregnancy on early mother to child transmission of HIV (MTCT) measured at 4-8 weeks postpartum in South Africa 2011-2012: a national population-based evaluation. PLoS ONE. 2015;10(5):e0125525.

2. Abdool Karim Q, Kharsany ABM, Frohlich JA, Werner L, Mlotshwa M, Madlala BT, et al. HIV incidence in young girls in KwaZulu-Natal, South Africa-public health imperative for their 
inclusion in HIV biomedical intervention trials. AIDS Behav. 2012;16(7):1870-6.

3. Johnson LF, Stinson K, Newell M-L, Bland RM, Moultrie H, Davies M-A, et al. The contribution of maternal HIV seroconversion during late pregnancy and breastfeeding to motherto-child transmission of HIV. J Acquir Immune Defic Syndr. 2012;59(4):417-25.

4. UNAIDS. Preventing mother to child transmission [Internet]. Available from https://www.unaids.org/en/keywords/preve nting-mother-child-transmission. Accessed 08 June 2021

5. Davey DJ, Farley E, Gomba Y, Coates T, Myer L. Sexual risk during pregnancy and postpartum periods among HIV-infected and -uninfected South African women: implications for primary and secondary HIV prevention interventions. PLoS ONE. 2018;13(3): 0 0192982.

6. Davey DJ, Farley E, Towriss C, Gomba Y, Bekker L-G, Gorbach $\mathrm{P}$, et al. Risk perception and sex behaviour in pregnancy and breastfeeding in high HIV prevalence settings: programmatic implications for PrEP delivery. PLoS ONE. 2018;13(5): 0197143.

7. Schwartz SR, Bassett J, Mutunga L, Yende N, Mudavanhu M, Phofa R, et al. HIV incidence, pregnancy, and implementation outcomes from the Sakh'umndeni safer conception project in South Africa: a prospective cohort study. Lancet HIV. 2019;6(7):e438-46.

8. Psaros C, Milford C, Smit JA, Greener L, Mosery N, Matthews LT, et al. HIV prevention among young women in South Africa: understanding multiple layers of risk. Arch Sex Behav. 2018;47(7):1969-82.

9. Fatti G, Shaikh N, Eley B, Jackson D, Grimwood A. Adolescent and young pregnant women at increased risk of mother-to-child transmission of HIV and poorer maternal and infant health outcomes: a cohort study at public facilities in the Nelson Mandela Bay Metropolitan district, Eastern Cape, South Africa. South Afr Med J Suid-Afr Tydskr Vir Geneeskd. 2014;104(12):874-80.

10. World Health Organization. Consolidated guidelines on the use of antiretroviral drugs for treating and preventing HIV infection [Internet]. World Health Organization; 2016. Available from http://www.who.int/hiv/pub/arv/arv-2016/en/. Accessed 21 Sep 2020

11. Davey DLJ, Pintye J, Baeten JM, Aldrovandi G, Baggaley R, Bekker L-G, et al. Emerging evidence from a systematic review of safety of pre-exposure prophylaxis for pregnant and postpartum women: where are we now and where are we heading? J Int AIDS Soc. 2020;23(1):e25426.

12. Heffron R, Mugo N, Hong T, Celum C, Marzinke MA, Ngure K, et al. Pregnancy outcomes and infant growth among babies with in utero exposure to tenofovir-based pre-exposure prophylaxis for HIV prevention. AIDS. 2018;32(12):1707-13.

13. Thigpen MC, Kebaabetswe PM, Paxton LA, Smith DK, Rose CE, Segolodi TM, et al. Antiretroviral preexposure prophylaxis for heterosexual HIV transmission in Botswana. N Engl J Med. 2012;367(5):423-34.

14. Molina J-M, Capitant C, Spire B, Pialoux G, Cotte L, Charreau I, et al. On-demand preexposure prophylaxis in men at high risk for HIV-1 infection. N Engl J Med. 2015;373(23):2237-46.

15. Grant RM, Lama JR, Anderson PL, McMahan V, Liu AY, Vargas $\mathrm{L}$, et al. Preexposure chemoprophylaxis for HIV prevention in men who have sex with men. N Engl J Med. 2010;363(27):2587-99.

16. Baeten JM, Donnell D, Ndase P, Mugo NR, Campbell JD, Wangisi $\mathrm{J}$, et al. Antiretroviral prophylaxis for HIV prevention in heterosexual men and women. N Engl J Med. 2012;367(5):399-410.

17. Baeten JM, Donnell D, Ndase P, Mugo NR, Campbell JD, Wangisi $\mathrm{J}$, et al. Antiretroviral prophylaxis for HIV-1 prevention among heterosexual men and women. N Engl J Med. 2012;367(5):399-410.
18. Stalter RM, Pintye J, Mugwanya KK. Safety review of tenofovir disoproxil fumarate/emtricitabine pre-exposure prophylaxis for pregnant women at risk of HIV infection. Expert Opin Drug Saf. 2021;28:1-7.

19. Joseph Davey DL, Pintye J, Baeten JM, Aldrovandi G, Baggaley R, Bekker L-G, et al. Emerging evidence from a systematic review of safety of pre-exposure prophylaxis for pregnant and postpartum women: where are we now and where are we heading? J Int AIDS Soc. 2020;23(1):e25426.

20. Koss CA, Bacchetti P, Hillier SL, Livant E, Horng H, Mgodi $\mathrm{N}$, et al. Differences in cumulative exposure and adherence to tenofovir in the VOICE, iPrEx OLE, and PrEP demo studies as determined via hair concentrations. AIDS Res Hum Retroviruses. 2017;33(8):778-83.

21. Kinuthia J, Pintye J, Abuna F, Mugwanya KK, Lagat H, Onyango $\mathrm{D}$, et al. Pre-exposure prophylaxis uptake and early continuation among pregnant and postpartum women within maternal and child health clinics in Kenya: results from an implementation programme. Lancet HIV. 2020;7(1):e38-48.

22. Stranix-Chibanda L, Anderson PL, Kacanek D, Hosek S, Huang S, Nematadzira TG, et al. Tenofovir diphosphate concentrations in dried blood spots from pregnant and postpartum adolescent and young women receiving daily observed pre-exposure prophylaxis in sub-Saharan Africa. Clin Infect Dis. 2020. https://doi.org/10. 1093/cid/ciaa1872.

23. Corneli A, Perry B, McKenna K, Agot K, Ahmed K, Taylor J, et al. Participants' explanations for nonadherence in the FEM-PrEP clinical trial. JAIDS J Acquir Immune Defic Syndr. 2016;71(4):452-61.

24. van der Straten A, Stadler J, Montgomery E, Hartmann M, Magazi B, Mathebula F, et al. Women's experiences with oral and vaginal pre-exposure prophylaxis: the VOICE-C qualitative study in Johannesburg, South Africa. PLoS One [Internet]. 2014; 9(2). Available from https://www.ncbi.nlm.nih.gov/pmc/articles/ PMC3931679/. Accessed 21 Sep 2020

25. O’Malley G, Barnabee G, Mugwanya K. Scaling-up PrEP delivery in Sub-Saharan Africa: what can we learn from the scale-up of ART? Curr HIV/AIDS Rep. 2019;16(2):141-50.

26. Pillay D, Stankevitz K, Lanham M, Ridgeway K, Murire M, Briedenhann E, et al. Factors influencing uptake, continuation, and discontinuation of oral PrEP among clients at sex worker and MSM facilities in South Africa. PLoS ONE. 2020;15(4):e0228620.

27. Kalichman SC, Shkembi B, Wanyenze RK, Naigino R, Bateganya MH, Menzies NA, et al. Perceived HIV stigma and HIV testing among men and women in rural Uganda: a population-based study. Lancet HIV [Internet]. 2020; 0(0). Available from https:// www.thelancet.com/journals/lanhiv/article/PIIS2352-3018(20) 30198-3/abstract. Accessed 16 Sep 2020

28. Earnshaw VA, Chaudoir SR. From conceptualizing to measuring HIV stigma: a review of HIV stigma mechanism measures. AIDS Behav. 2009;13(6):1160-77.

29. Brooks RA, Landrian A, Nieto O, Fehrenbacher A. Experiences of anticipated and enacted pre-exposure prophylaxis (PrEP) stigma among Latino MSM in Los Angeles. AIDS Behav. 2019;23(7):1964-73.

30. Velloza J, Khoza N, Scorgie F, Chitukuta M, Mutero P, Mutiti K, et al. The influence of HIV-related stigma on PrEP disclosure and adherence among adolescent girls and young women in HPTN 082: a qualitative study. J Int AIDS Soc. 2020;23(3):e25463.

31. Zuch M, Mason-Jones AJ, Mathews C, Henley L. Changes to the law on consent in South Africa: implications for school-based adolescent sexual and reproductive health research. BMC Int Health Hum Rights. 2012;12(1):3.

32. Statement by President Cyril Ramaphosa on measures to combat COVID-19 epidemic. I The Presidency [Internet]. Available from 
/press-statements/statement-president-cyril-ramaphosa-measurescombat-covid-19-epidemic. Accessed 08 Oct 2020

33. National Department of Health. National HIV counselling and testing policy guidelines [Internet]. 2015. Available from https:// www.nicd.ac.za/assets/files/HCT-Guidelines-2015.pdf. Accessed 14 Sep 2020.

34. Harris PA, Taylor R, Minor BL, Elliott V, Fernandez M, O'Neal $\mathrm{L}$, et al. The REDCap consortium: building an international community of software platform partners. J Biomed Inform. 2019;95:103208.

35. Harris PA, Taylor R, Thielke R, Payne J, Gonzalez N, Conde JG. Research electronic data capture (REDCap) - A metadata-driven methodology and workflow process for providing translational research informatics support. J Biomed Inform. 2009;42(2):377-81.

36. National Department of Health. Sexually transmitted infections: management guidelines 2018 [Internet]. 2018. Available from https://www.nicd.ac.za/wp-content/uploads/2019/10/STI-Guide lines-27-08-19-1Final.pdf. Accessed 18 Sep 2020.

37. Pyra M, Anderson P, Haberer JE, Heffron R, Celum C, Asiimwe $\mathrm{S}$, et al. Tenofovir-diphosphate as a marker of HIV pre-exposure prophylaxis use among east African men and women. Front Pharmacol. 2019;10:401.

38. Bekker L-G, Rebe K, Venter F, Maartens G, Moorhouse M, Conradie F, et al. Southern African guidelines on the safe use of pre-exposure prophylaxis in persons at risk of acquiring HIV-1 infection. South Afr J HIV Med. 2016;17(1):11.

39. National Department of Health. Guidelines for the provision of Pre-exposure prophylaxis (PrEP) to persons at substantial risk of HIV infection [Internet]. National Department of Health, Republic of South Africa; 2020. Available from https://www.prepwatch.org/ resource/south-african-guidelines-prep-2020/. Accessed $21 \mathrm{Apr}$ 2021

40. AUDIT : the alcohol use disorders identification test : guidelines for use in primary health care [Internet]. Available from https:// www.who.int/publications-detail-redirect/audit-the-alcohol-usedisorders-identification-test-guidelines-for-use-in-primary-healthcare. Accessed 18 Sep 2020

41. Berman AH, Bergman H, Palmstierna T, Schlyter F. DUDIT. Drug Use Disord Identif Test-E Man Karolinska Institutet Stockh. 2007

42. Basile KC, Hertz MF, Black SE. Intimate partner violence and sexual violence victimization assessment instruments for use in healthcare settings. Version 1. 2007
43. Garcia-Moreno C, Jansen HA, Ellsberg M, Heise L, Watts CH, et al. Prevalence of intimate partner violence: findings from the WHO multi-country study on women's health and domestic violence. Lancet. 2006;368(9543):1260-9.

44. Pintye J, Davey DLJ, Wagner AD, John-Stewart G, Baggaley R, Bekker L-G, et al. Defining gaps in PrEP delivery for pregnant and breastfeeding women in high burden settings using an implementation science framework. Lancet HIV. 2020;7(8):e582-92.

45. IMPAACT 2009 I IMPAACT [Internet]. Available from https:// www.impaactnetwork.org/studies/impaact2009. Accessed 22 May 2021

46. Lawrie TA, Hofmeyr GJ, de Jager M, Berk M. Validation of the Edinburgh postnatal depression scale on a cohort of South African women. S Afr Med J. 1998;88(10):1340-4.

47. R Core Team. R: A language and environment for statistical computing [Internet]. Vienna, Austria: R foundation for statistical computing; 2020. Available from https://www.R-project.org/. Accessed 12 Sep 2020.

48. Vazquez L, Moll AP, Kacin A, Ndlovu NE, Shenoi SV. Perceptions of HIV preexposure prophylaxis among young pregnant women from Rural KwaZulu-Natal, South Africa. AIDS Patient Care STDs. 2019;33(5):214-9.

49. Davey DLJ, Bekker L-G, Mashele N, Gorbach P, Coates TJ, Myer L. PrEP retention and prescriptions for pregnant women during COVID-19 lockdown in South Africa. Lancet HIV [Internet]. 2020; 0(0). Available from https://www.thelancet.com/journals/ lanhiv/article/PIIS2352-3018(20)30226-5/abstract. Accessed 19 Oct 2020

50. Davey DJ, Myer L, Coates T. PrEP implementation in pregnant and postpartum women. Lancet HIV. 2020;7(1):e5-6.

51. Joseph Davey DL, Knight L, Markt-Maloney J, Tsawe N, Gomba Y, Mashele N, et al. I had Made the Decision, and No One was Going to Stop Me-Facilitators of PrEP adherence during pregnancy and postpartum in Cape Town, South Africa. AIDS Behav. 2021. https://doi.org/10.1007/s10461-021-03320-x.

52. South Africans prepare for three-week lockdown. BBC News [Internet]. Available from https://www.bbc.com/news/av/worldafrica-52055161. Accessed 08 Oct 2020

Publisher's Note Springer Nature remains neutral with regard to jurisdictional claims in published maps and institutional affiliations. 